\title{
Leprosy elimination campaigns: the Nigerian experience
}

\author{
O. SOFOLA \\ National Tuberculosis and Leprosy Control Programme, Federal \\ Ministry of Health, Department of Primary Health Care and Disease \\ Control, Federal Secretariat, Ikoyi, Lagos, Nigeria
}

Accepted for publication 14 October 1999

\section{Introduction}

Leprosy is a major public health problem in Nigeria. The National Tuberculosis and Leprosy Control Programme (NTBLCP) was established in 1989 and formally launched in 1991 when we commenced nationwide implementation of MDT. The LEC programme in Nigeria is shown in Figure 1.

In 1989, Nigeria had about 200,000 registered leprosy cases. ${ }^{1}$ Since then, the leprosy situation in Nigeria has improved significantly. The programme enjoys financial and technical support from members of the International Federation of Anti-Leprosy Associations and the World Health Organization (WHO).

LEPROSY SITUATION IN NIGERIA - 1998

Registered prevalence

Prevalence rate

Detection rate

Disability grade 2 among new cases

Proportion of MB cases

MDT coverage

Cumulative no. cured on MDT
7534

$0 \cdot 6$ per 10,000 population

5.9 per 100,000 population

$14 \%$

$74 \%$

$100 \%$

64,419

Although Nigeria has achieved the elimination target of under 1 case per 10,000 population at the national level, there are endemic pockets within the country. Ten out of the 36 States and the capital have prevalence rates between 1 and 2 per 10,000 population. Furthermore, leprosy control has been predominantly organized as a vertical programme with a network of MDT clinics all over the country. Only recently have MDT services been integrated within the general health services.

In view of the high disability rate (14\%) and high MB proportion (74\%) among new cases indicating late detection of cases, we felt that we may have yet undetected and untreated cases in some areas of the country. ${ }^{2}$ 


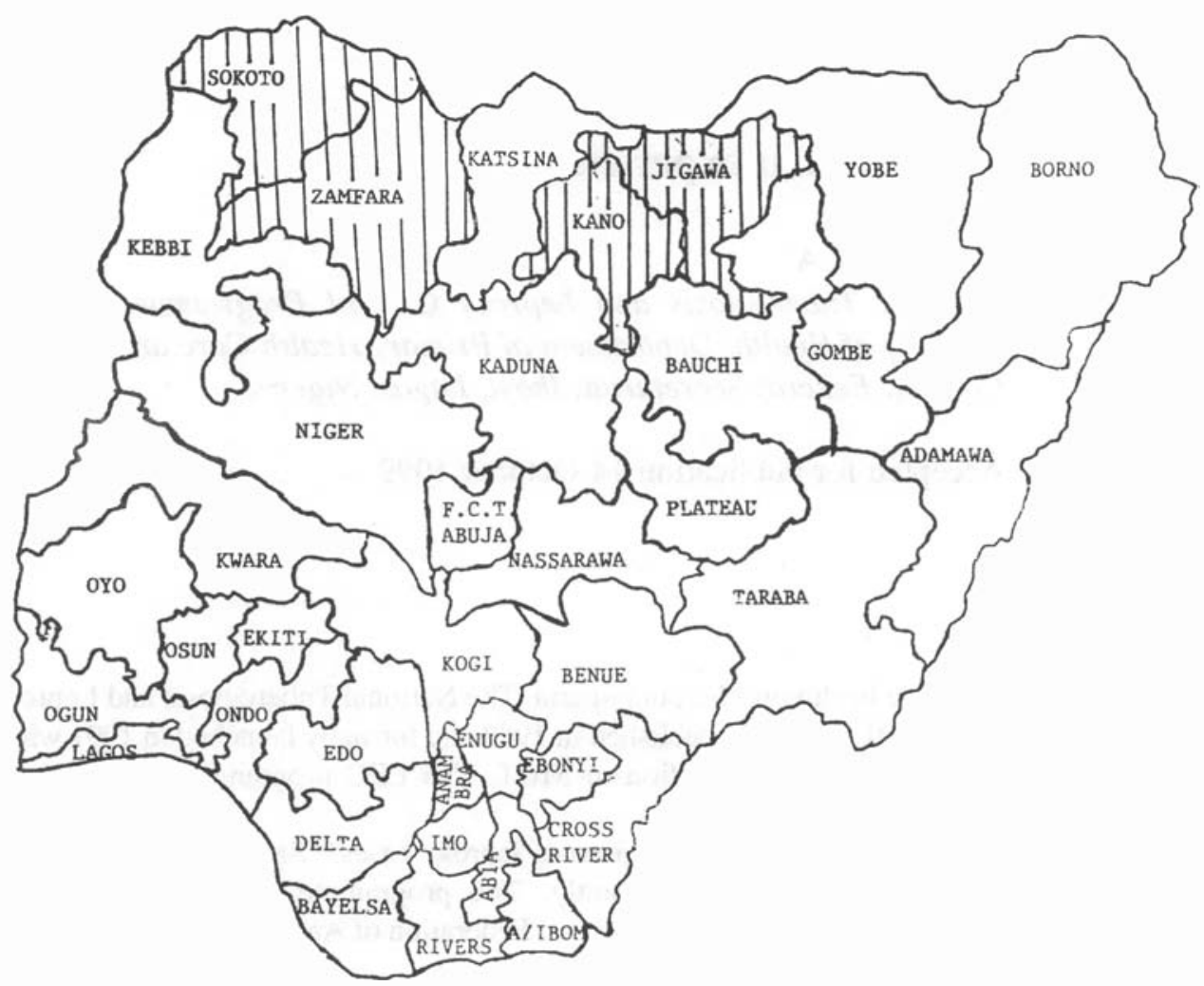

Figure 1. Map of Nigeria, showing LEDC areas (shaded).

In 1996, Nigeria embarked on the WHO initiated Leprosy Elimination Campaign (LEC) in order to address the issue of hidden leprosy cases and with the aim of achieving the elimination target by the year 2000, not only at the national level but also at the sub-national level. LEC is a one-time action support to strengthen existing leprosy services and can be repeated if necessary.

\section{OBJECTIVES OF LEPROSY ELIMINATION IN NIGERIA}

1. To detect by passive case-finding and treat hidden leprosy cases, especially the multibacillary cases in the communities selected.

2. To increase community awareness through mass public enlightenment in order to increase the number of patients self-reporting.

3. To open new clinics in the selected areas with a view to expanding geographical coverage and facilitating integration of leprosy services within general health services.

In order for LECs to have an impact on leprosy elimination and also be cost-effective, 
they need to cover large populations and must be able to detect and cure most of the hidden cases within a relatively short period of time. ${ }^{3}$

To achieve this, the last four LECs in Nigeria were carried out to cover four endemic States of the country namely Sokoto, Zamfara, Kano and Jigawa.

The criteria for selection of LEC area according to WHO guidelines include easy accessibility of villages/communities, availability of health infrastructure and health staff to ensure continuity. Other factors are high prevalence compared with other areas and the majority of new patients should be MB cases with more than $10 \%$ with grade 2 disability at presentation. ${ }^{4}$

\section{Leprosy situation in project area before campaign}

In Sokoto, Zamfara, Kano and Jigawa States, all registered cases have been out on MDT. ${ }^{5}$ The proportion of MB cases among new cases in high (75-85\%) and the disability grade 2 is high $(11-31 \%)$.

Leprosy activity is mainly vertical. The number of MDT clinics in the areas varies between 15 and $30 \%$ of health facilities available in these areas. Table 1 shows a summary of leprosy situation in the areas before LEC.

\section{Main activities carried out during LEC}

Advocacy visits were carried out by the state leprosy control officer and the central unit staff to State Ministries of Health, Local Government Authorities, Heads of Village Committees and Community Heads. The objective of the meeting was to secure political and administrative support for LEC.

Radio jingles produced in local languages, stressing the signs, symptoms and curability of leprosy and informing community members that special teams would be in their areas to put proven cases on treatment, were aired over a 10-week period (before and during casefinding). This coincided with prime periods of broadcasting to ensure extensive coverage of the populace. Posters and flyers were also distributed. Mobile vehicles with loud speakers were used during market days as well as town criers to pass on information to the villagers about the team's arrival including date and venue.

Table 1. Leprosy situation of the areas before LEC

\begin{tabular}{lcrr}
\hline & Sokoto/Zamfara & Kano & Jigawa \\
\hline Commencement of statewide leprosy control activities & 1993 & 1991 & 1991 \\
MDT coverage (\%) & 100 & 100 & 100 \\
Registered prevalence 1997 & 778 & 1530 & 1657 \\
New cases 1997 & 471 & 475 & 532 \\
Proportion of MB cases among new cases (\%) & 75 & 85 & 80 \\
Disability grade 2 among new cases (\%) & 31 & $10 \cdot 9$ & 24 \\
No. of MDT clinics & 170 & 80 & 61 \\
No. of health facilities & 581 & 551 & 304 \\
\end{tabular}


Training workshops for the implementers of LEC were organized. A 1-day workshop for trainers (TOT), targeted at members of the special teams, was conducted by the Programme Manager and WHO Consultant. Members of the Supervisory and Monitoring teams also attended the workshop. The participants were briefed on the disease and control measures. WHO guidelines to train general health workers and volunteers were highlighted. ${ }^{6}$

The TOT workshop was followed by a 1-day training workshop (in the morning) for identified general health workers in all the health centres in the areas, as well as the volunteers (in the evening of the same day). The workshops were carried out in zones and facilitated by State Leprosy Control Officer and some participants at the TOT workshop.

Primarily, the workshops were aimed at equipping the participants with the basic knowledge and the signs and symptoms of leprosy, participants were also informed on proper dissemination of information to the rural populace for adequate mobilization.

Case-finding activities were carried out over a period of 2-3 months. The special teams travelled from village to village according to the itineraries. Their arrival at the villages was preceded by announcement by village town criers and mobilization by volunteers. Upon arrival of the special team, the chief or town crier helped to summon the villagers to the venue where they were given health education on leprosy. All persons, especially those with skin lesions, were encouraged to be examined either in makeshift huts, school buildings or at the health facilities. Proven leprosy cases were started on treatment with MDT and documented. The special teams comprised trained leprosy control supervisors, general health staff and sometimes a voluntary village worker.

The supervisory teams monitored progress of the campaign, re-examined skin lesions that posed problem for the special teams and solved logistic problems. However, the use of trained leprosy supervisors for case-finding minimized wrong-diagnosis.

\section{Achievements}

The results are shown in Table 2. Most (85-90\%) of communities in the project areas were visited. The number of villagers examined represented about $2-9 \%$ of the population of the areas. However, a much higher proportion of the population was health educated, both

Table 2. Results of LEC in Sokoto, Zamfara, Kano and Jigawa states

\begin{tabular}{lllll}
\hline & Sokoto & Zamfara & Kano & Jigawa \\
\hline Population & $2,764,442$ & $2,115,595$ & $6,700,000$ & $3,472,000$ \\
No. of suspects & 56,117 & 104,000 & 346,387 & 347,039 \\
No. of villages screened & 383 & 302 & 1000 & 747 \\
No. of new cases & 227 & 126 & 476 & 927 \\
On MDT & 87 & 31 & 93 & 148 \\
$\quad$ PB & $140(61)$ & $95(75)$ & $383(80)$ & $774(84)$ \\
$\quad$ MB (\%) & $3 \cdot 3$ & $9 \cdot 6$ & $7 \cdot 9$ & $31 \cdot 3$ \\
Proportion of children (\%) & $16 \cdot 3$ & 27 & $13 \cdot 8$ & $6 \cdot 5$ \\
Proportion of disability grade 2 (\%) & 293 & 229 & 304 & 567 \\
No. of local health workers trained & 191 & 117 & 368 & 550 \\
No. of volunteers trained & 45 & 11 & 243 & 487 \\
No. of new MDT clinics opened & & & \\
\hline
\end{tabular}


through the mass media and the health talks given to the villagers from village to village. The total number of new cases found during LEC in the project areas was 1731. The proportion with grade 2 disability varied between 6.5 and $27 \%$. The MB proportion was high $(61-84 \%)$, which is similar to what obtains in our routine leprosy services. The case detection rose from $5 \cdot 9$ to $12 / 100,000$.

A total of 1915 general health workers and 1226 volunteers were trained, thus improving MDT services expertise in those areas. A total of 786 new clinics were opened, which improved accessibility of MDT services to patients. In Sokoto and Zamfara States, only new clinics with patients were opened while in Kano and Jigawa, all the PHC facilities are being utilized for MDT services. The latter is in line with WHO recommendation.

\section{Problems encountered and solutions}

1. Frequent breakdown of some of the motorcycles used during the project because of the large expanse of area covered. The NGOs provided financial support to effect repairs quickly.

2. Some villages could not be visited because of tribal clashes. It is hoped that such villages will be visited later.

3. The half-day allocated to training of general health workers according to LEC guidelines was not adequate to cover more aspects of leprosy control in view of integration currently being pursued in the programme and the tasks allocated to general health workers. To improve the situation, NGOs are willing to pay for accommodation for 1 day to extend the training period to $1 \frac{1}{2}$ days.

4. In some municipalities (cities), individuals were reluctant to come forward to be examined in the venues ear marked for that purpose. However, those with skin lesions who had been sensitized through radio jingles and posters later reported to the leprosy Referral Hospital in the locality.

\section{Sustaining activities}

The following activities will be carried out to sustain the gains of LEC:

- Routine and regular supervision to be undertaken by State Leprosy Control Officer and TBL Supervisors to support field staff.

- Adequate provisions of MDT drugs will be made available in the treatment centres.

- Periodic (re) orientation training of general health workers to encourage integration of leprosy services within general health services.

- Screening of contacts.

- Continue close working relationship with donors for both financial and logistics support.

- Maintain political commitment at all levels of government.

\section{Impact of LEC on the national programme}

Low detection due to poor awareness about leprosy and unavailability of MDI services in some areas of the States of the country was a major problem in the national programme. 
COMPARISON OF THE LEPROSY SITUATION IN L.E.C. AREAS, NEW CASES DETECTED

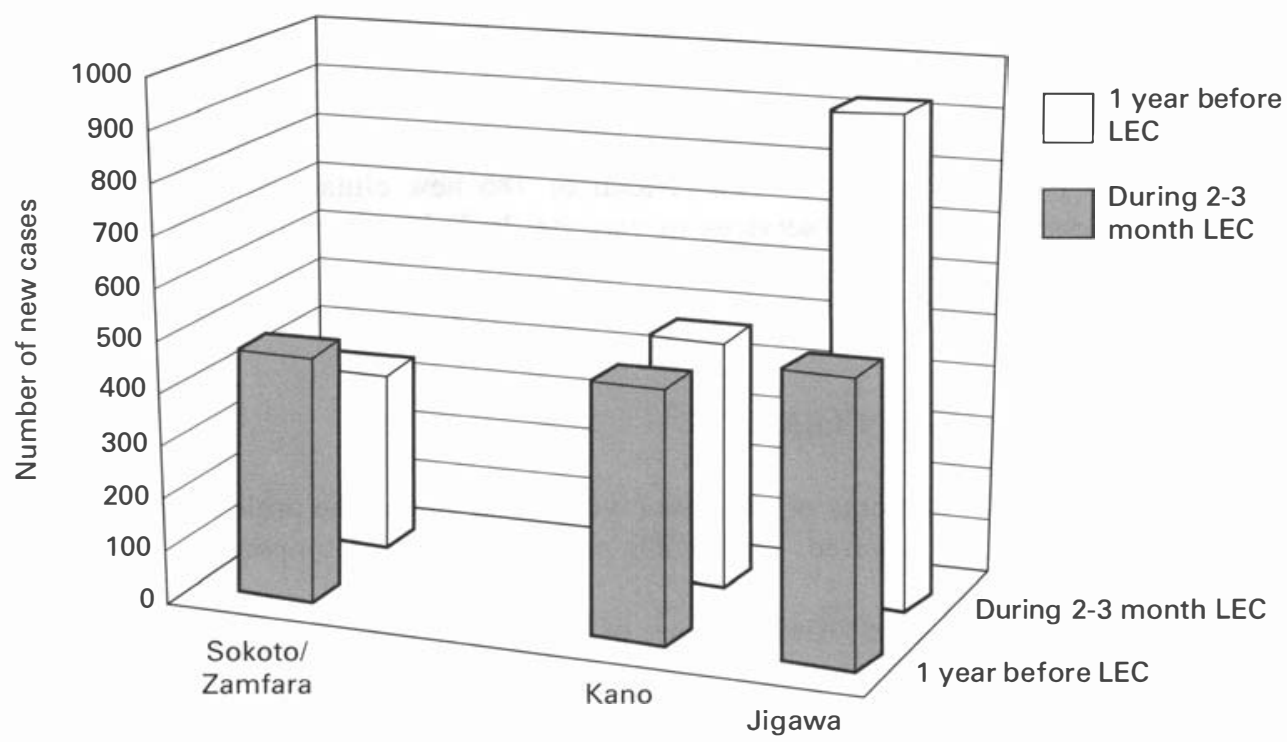

States in which LEC were carried out

Figure 2. Comparison of the leprosy situation in LEC areas: new cases detected.

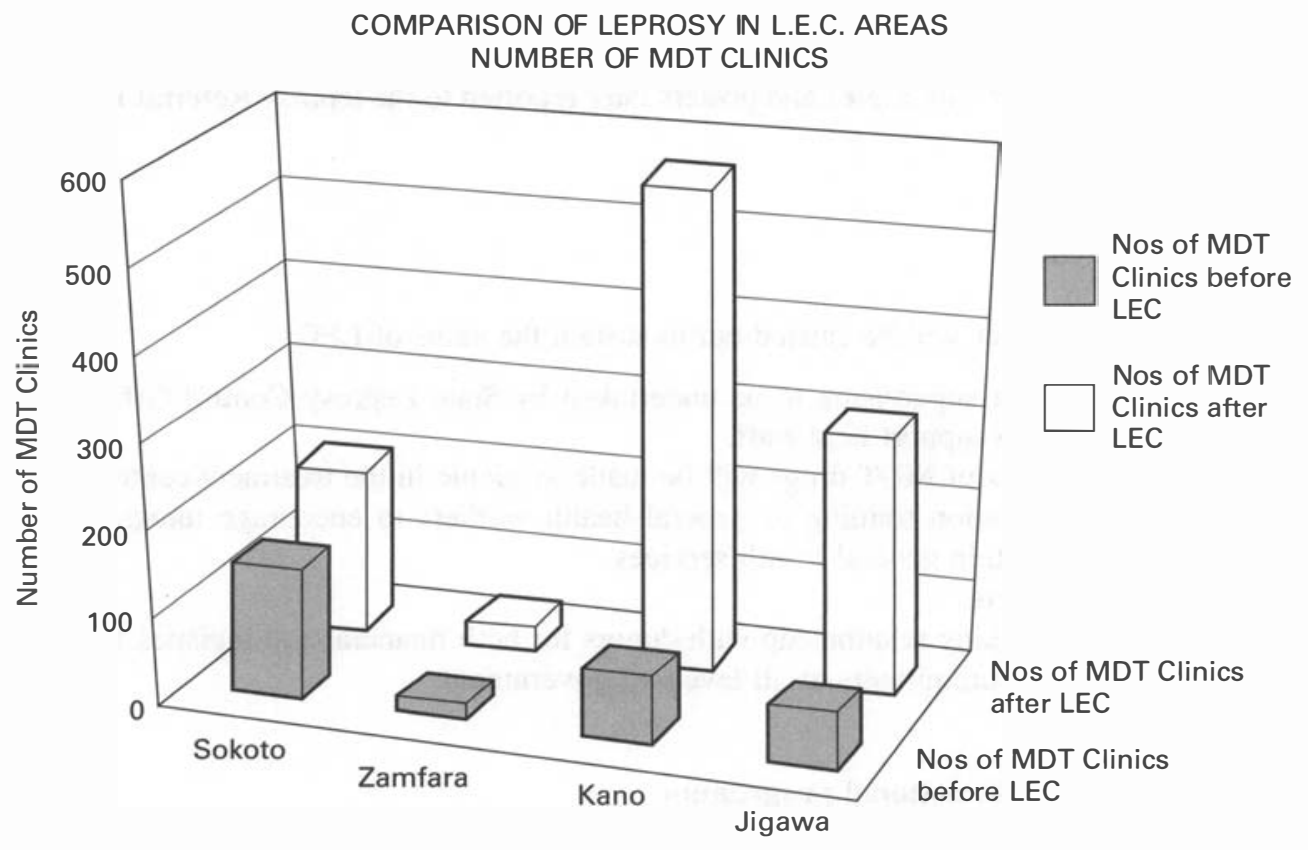

States in which LEC were carried out

Figure 3. Comparison of leprosy situation in LEC areas: number of MDT clinics. 
Through LEC, a significant proportion of hidden cases was detected in the project areas, thus improving case detection in the national programme. It is expected that following LEC, detection will stabilize and then reduce. Figure 2 shows a chart of cases detected 1 year before LEC and those detected within the short period (2-3 months) of LEC.

LEC has also made MDI services more accessible to patients in these areas. The number of MDT clinics increased from 311 to 1097 after LEC. Figure 3 shows a comparison of number of MDI clinics in the project areas before and after LEC. It is expected that through the availability of trained and motivated health workers during LEC most of the health facilities in the areas will be capable of providing MDT services.

\section{Conclusion}

In Nigeria, LEC has been found to be a useful intervention to improve case detection and to facilitate the integration of leprosy services within the general health services. LEC has indeed strengthened our routine leprosy services and enables us to involve new partners general health workers and volunteers in the fight against leprosy.

Therefore, their continuation in other selected areas of the country is most justified to complete and sustain leprosy elimination activities at the sub-national level.

\section{References}

1 Workers manual, Federal Ministry of Health, Nigeria.

2 National Tuberculosis and Leprosy Control Programme (NTBLCP) Annual Report, 1998.

3 World Health Organization. Weekly Epidemiological Record, 1998, 24; 177-184.

4 World Health Organization. WHO guidelines for carrying out leprosy elimination campaigns. WHO, Geneva, 1996.

5 NTBLCP Annual Report, 1997.

${ }^{6}$ World Health Organization. A guide to eliminating leprosy. WHO, Geneva, 1995. 